\title{
Caring for the carer: Are we doing enough?
}

\author{
S R Perera, T P M Fonseka, D K Amarasinghe, S S Williams
}

\section{Abstract}

In Asian societies, the family carer has a pivotal role in ensuring the wellbeing of a person with mental health needs. In the absence of adequate mental health professionals and services, they take on the responsibility of identifying any changes in emotions and behaviour, convincing the person to seek medical help, accompanying the person on the visits to the doctor, and ensuring treatment compliance, follow up and rehabilitation. They have to buffer the stigma, find the financial resources and even manage the risks. In such situations, the burden on the carer is manifold. The physical and emotional strain can be enormous. Therefore, the treating physicians have to go out of their way to support the carer and ensure that they are adequately educated, supported and comforted on their journey of caregiving. Without their partnership, the care of the patient is destined to fail.

\section{Introduction}

Sir Anthony Hopkins wins another Oscar in 'The Father' at 83 years, portraying a person with dementia. The film not only highlights the struggles of a person with dementia but also that of his daughter Anne, played by Olivia Colman, who too was nominated for the best supporting actress. Coming to terms with and coping with mental and behavioural disorders no doubt is very challenging for family carers.

In Asian societies, by default, most of the care for a person with a serious mental disorder is provided by a family carer, apart for short hospital admissions that may sometimes be arranged during acute crisis situations. Institutions with longer stay facilities are few and far between, are costly and often exploitative in nature. Formal community care is a pipe dream. In this context, the onus of caregiving falls on immediate family, often females - mothers, wives, daughters or sisters. The demands on these carers are insurmountable.

\section{Terminology}

A carer as discussed here is a person who provides some type of unpaid, ongoing assistance during a mental health crises, subsequent treatment and recovery. The carer is typically a family member or a friend who attends to the physical, emotional and sometimes even financial needs of the person (1). They may or may not live in the same household and usually do not have fixed hours or terms or conditions of employment. This service is in contrast to formal caregiving provided by health care professionals or paid carers.

\section{Burden on the carer}

When a person develops a mental disorder, restrictions are imposed on the family out of the blue. This is especially true for the person in the family who takes on the role of the main carer (2). The main carer often unwittingly has to continue in this role for many years (3). It has to be emphasized that the burden on the carer of a person with a mental disorder is higher than for carers of those with chronic medical conditions (4).

Relapses prove to be unsettling for family relationships, work and life in general. The fact that certain symptoms remain even after the patient has been discharged from hospital or while he/she is in remission, demands a longerterm commitment from the family carer.

Carer stress is associated with physical health complaints and increased use of primary health care services. Carers may develop maladaptive coping and deficient health practices that contribute to poor physical and mental health (5). Some carers use avoidance coping, show emotional reactivity and wishful thinking (6). A higher burden is also perceived when carers have no control over the patient's challenging and problem behaviours 
(7). Research shows that carers have higher rates of depression, anxiety and substance misuse, compared to the normal population (8). Studies done amongst carers of patients with dementia found that one in three carers had depression, which is much higher than in their counterparts who were caring for patients with other medical illnesses (9). Interestingly, depression is more common among female carers, carers with male care recipients and in carers who have a spousal relationship with the patient (10).

The issue of stigma is all consuming and the family and especially the carers, have to face it day in and day out (11). This is compounded by financial burden, loss of recreation and free time, carer guilt and burnout (12-14). Caught up in their role as carers, they sacrifice their sleep, exercise, health visits and social engagements in the provision of support to their patient.

\section{Perspectives of the carer}

A descriptive study in Iran showed that social support, emotional support, safety and security were the most important supportive factors that family carers requested. These carers benefitted from the support given by other family members, neighbours and religious leaders, although they lacked respite care that high income countries offered (15). A qualitative study in Malaysia found that carers used religious coping, emotional coping and acceptance as coping strategies. Leisure activities and the use of traditional healing methods too were perceived as helpful (16). Social support and support from the family itself improved the relationships within the family, as well as with the ill relative (17).

The Asian philosophy from ancient times is based on caregiving for the infirm. Most religions promote the benefits of caring for the vulnerable and its benefits in the present and the afterlife. As such, a failure to provide satisfactory care could in itself cause guilt in the caregiver.

\section{Caring for the carer}

Considering the magnitude of the struggles of the carer, the importance of caring for the carer, from the first visit to the mental health service provider, cannot be overemphasized. It is the bounden duty of the doctor to always provide a private opportunity for the carer to discuss their concerns, provided the patient has no objection. It is questionable whether such a formal protocol is in place even in the most advanced of mental health facilities.

Numerous scales have been developed to measure carer burden and various strategies have been implemented to alleviate carer burden and suffering. Studies reveal that targeted interventions can reduce family members' burden, improve knowledge of the disease and treatment, and increase their ability to cope (18). Provision of direct support to the carers, ranging from psychological support to training and respite care have been discussed (1). Similarly, support and peer groups too are of value. However, in reality most carers do not have the resources, including the time and money, to access these support mechanisms, even if available.

As such, every meeting with the patient should be an opportunity to inquire about the carer. Meeting the needs of the carer will directly and indirectly impact on the wellbeing of the patient. Both the emotional and physical needs of the caregiver have to be addressed. We need more research on the possible increased morbidity and mortality of carers due to neglect of their physical health. Holistic interventions that address carer health, including acknowledgement of the role of religious and cultural practices, have to be developed and manualized.

\section{Conflicts of interest}

None declared.

S R Perera, Department of Psychiatry, Faculty of Medicine, University of Peradeniya, Sri Lanka

T P M Fonseka, North Colombo Teaching Hospital, Sri Lanka

D K Amarasinghe, Teaching Hospital, Peradeniya, Sri Lanka

S S Williams, Department of Psychiatry, Faculty of Medicine, University of Kelaniya, Sri Lanka

Corresponding author: S S Williams

Email: shehanwil@gmail.com

http://orcid.org/0000-0001-7764-5550

\section{References}

1. Parks SM, Novielli KD. A practical guide to caring for caregivers. Am Fam Physician 2000; 62: 2613-22.

2. Zarit SH, Reever KE, Bach-Peterson J. Relatives of the impaired elderly: correlates of feelings of burden. Gerontologist 1980; 20: 649-55.

3. Cheng ST. Dementia Caregiver Burden: a Research Update and Critical Analysis. Curr Psychiatry Rep 2017; 19: 64.

4. Ampalam P, Gunturu S, Padma V. A comparative study of caregiver burden in psychiatric illness and chronic medical illness. Indian J Psychiatry 2012; 54: 239-43.

5. Scazufca M, Kuipers E. Coping strategies in relatives of people with schizophrenia before and after psychiatric admission. Br J Psychiatry 1999; 174: 154-158. 
6. Rigby T, Ashwill RT, Johnson DK, Galvin JE. Differences in the Experience of Caregiving Between Spouse and Adult Child Caregivers in Dementia With Lewy Bodies. Innov Aging 2019; 4: 3-4.

7. Sallim AB, Sayampanathan AA, Cuttilan A, Ho R. Prevalence of Mental Health Disorders Among Caregivers of Patients With Alzheimer Disease. J Am Med Dir Assoc 2015; 16: 1034-41.

8. Katon W. Depression: relationship to somatization and chronic medical illness. J Clin Psychiatry 1984; 45: 4-12.

9. Covinsky KE, Newcomer R, Fox P, et al. Patient and caregiver characteristics associated with depression in caregivers of patients with dementia. J Gen Intern Med 2003; 18: 1006-14.

10. Adams KB. Specific effects of caring for a spouse with dementia: differences in depressive symptoms between caregiver and non-caregiver spouses. Int Psychogeriatr 2008; 20: 508-20.

11. Werner P, Mittelman MS, Goldstein D, Heinik J. Family stigma and caregiver burden in Alzheimer's disease. Gerontologist 2012; 52: 89-97.

12. Coyne JC, Kessler RC, Tal M, Turnbull J, Wortman CB, Greden JF. Living with a depressed person. J Consult Clin Psychol 1987; 55: 347-52.
13. Corrigan P. How stigma interferes with mental health care. Am Psychol 2004; 59: 614-25.

14. Kasuya RT, Polgar-Bailey P, Takeuchi R. Caregiver burden and burnout. A guide for primary care physicians. Postgrad Med 2000; 108: 119-23.

15. Akbari M, Alavi M, Irajpour A, Maghsoudi J, Lopez V, Cleary M. Support Needs for Family Caregivers of Clients with Mental Illness in Iran: A Qualitative Study. Issues Ment Health Nurs 2018; 39: 896-903.

16. van Vun JS, Cheah WL, Helmy H. Mental Health Status and Its Associated Factors Among Caregivers of Psychiatric Patients in Kuching, Sarawak. Malays Fam Physician 2019; 14: 18-25.

17. Hsiao CY. Family demands, social support and caregiver burden in Taiwanese family caregivers living with mental illness: the role of family caregiver gender. J Clin Nurs 2010; 19: 3494-503.

18. Yesufu-Udechuku A, Harrison B, Mayo-Wilson E, et al. Interventions to improve the experience of caring for people with severe mental illness: systematic review and meta-analysis. Br J Psychiatry 2015; 206: 268-74. 